\title{
Dataset: Feedback on the Path2Integrity learning cards for research integrity
}

\author{
Lisa Häberlein $¥$, Oliver Claas $\ddagger$ \\ ‡ Coburg University, Coburg, Germany
}

Corresponding author: Lisa Häberlein (lisa.haeberlein@hs-coburg.de)

Reviewable v1

Received: 07 Sep 2020 | Published: 10 Sep 2020

Citation: Häberlein L, Claas O (2020) Dataset: Feedback on the Path2Integrity learning cards for research integrity. Research Ideas and Outcomes 6: e58434. https://doi.org/10.3897/rio.6.e58434

\section{Abstract}

This dataset lists feedback given by participants and educators of lessons based on the Path2Integrity learning cards for research integrity. The feedback recorded both positive and negative teaching experiences, what worked well and which difficulties the educators encountered. It is deemed relevant for the further improvement of the learning cards.

\section{Keywords}

Path2Integrity learning cards; Teaching research integrity; Path2Integrity session; Path2Integrity workshop; Teaching experience

\section{Description of the dataset}

This dataset lists feedback given by participants and educators of lessons based on the Path2Integrity learning cards for research integrity (Suppl. material 1). The Path2Integrity learning cards, which promote knowledge and competence leading to reliable research results, have been used continuously since the release of their first version in September 2019. The feedback presented in this collection was given to the authors and co-authors of the learning cards by selected and experienced teachers and trainers with various professional and academic backgrounds. These educators have familiarised themselves thoroughly with the materials and shared their insights after each session. The feedback 
recorded both positive and negative teaching experiences, what worked well and which difficulties the educators encountered. It is deemed relevant for the further improvement of the learning cards.

Each row of the dataset consists of a number, the first digit of which refers to the session, the second digit to the comment in that session; then the comment; the number of the learning card; the version of the learning card; the educational level of the audience which worked with the card; the audience size; and the year and the country in which the session was held.

Authors and co-authors have used the feedback to improve the learning cards as well as the Path2Integrity handbook of instructions for teachers and trainers. While the original learning cards are in English, some of the sessions were held in a different language. This generated linguistic recommendations which will be used in the future translation of the learning material into other European languages.

The dataset will be updated with feedback from teaching activities until the final version of the Path2Integrity learning cards is completed. This allows for the transparent documentation of the development and improvement of the Path2Integrity learning cards.

\section{Funding program}

This project receives funding from the European Union's Horizon 2020 research and innovation programme under grant agreement No 824488.

\section{Grant title}

Path2Integrity - Rotatory role-playing and role-models to enhance the research integrity culture

\section{Hosting institution}

Coburg University of Applied Sciences and Arts, Coburg, Germany

\section{Supplementary material}

\section{Suppl. material 1: Dataset: Feedback on the Path2Integrity learning cards for research integrity doi}

Authors: Julia Prieß-Buchheit; Lisa Häberlein; Oliver Claas

Data type: Feedback

Download file (44.78 kb) 